\title{
Invasive Procedures of the Chest Lesions-Are they must be Performed and Why
}

\author{
Atanas Hilendarov ${ }^{1 *}$, Alexandar Georgiev ${ }^{2}$, Lyubomir Chervenkov ${ }^{3}$ \\ ${ }^{1}$ Associate Professor, Department of Diagnostic Imaging, Medical University, Plovdiv, Bulgaria \\ ${ }^{2}$ Researcher, Department of Diagnostic Imaging, Medical University, Plovdiv, Bulgaria \\ ${ }^{3}$ Radiologist, Department of Diagnostic Imaging, Medical University, Plovdiv, Bulgaria
}

*Address for Correspondence: Dr. Hilendarov Atanas, Associate Professor, Department of Diagnostic Imaging, MU Plovdiv 49 Volga str, ap.14, 6 fl, 4002 Plovdiv, Bulgaria

E-mail: dr hill@abv.bg

Received: 26 Dec 2020/ Revised: 23 Feb 2021/ Accepted: 23 Apr 2021

\begin{abstract}
Background: Chest tumours, in particular lung cancer, remain one of the most common causes of death worldwide. Using MD spiral CT, an increasing number of lung and mediastinal lesions is detected and histological diagnosis is often necessary to determine the most appropriate management of these lesions.

Methods: Fine-needle aspiration biopsy (FNAB) and core-needle biopsy (CNB) currently is the predominant method for obtaining tissue specimens in patients with lung lesions. In many cases, treatment protocols are based on histological information. In 85 of all 97 patients included in ours study FNAB biopsy is performed and in 12 CNB, when technically feasible, or in cases where other techniques (such as bronchoscopy with lavage) are inconclusive. The 19-22G disposable needles were used.

Results: In all 76 patients, aged 21-79 years with pulmonary lesions with dimensions of $2.0 \mathrm{~cm}$ or less FNB under CT control are performed. In 13 cases FNA under US control is performed due to the superficial localization of the lesions. Cytological and evaluation of FNAB samples were performed in all patients. Diagnostic sensitivity and accuracy are calculated. Assess the type of complications that occurred. FNAB and CNB, with the latter, demonstrated to have a slightly higher overall sensitivity, specificity and accuracy.

Conclusion: Percutaneous FNAB and CNB are safe procedures even though a few complications are possible: pneumothorax, pulmonary haemorrhage is common, while air embolism and seeding are rare, but with severe consequences.
\end{abstract}

Key-words: Core-needle biopsy, Diagnostic accuracy, Fine-needle aspiration biopsy, Lung lesion

\section{INTRODUCTION}

Chest tumours, in particular lung cancer, remain one of the most common causes of death worldwide. Using MD spiral CT, an increasing number of lung and mediastinal lesions is detected and histological diagnosis is often necessary to determine the most appropriate management of these lesions ${ }^{[1]}$. Various imaging techniques including computed tomography (CT) fluoroscopy and ultrasound (US) can be used to guide chest biopsies. CT is the most frequently used methods because of its high spatial and contrast resolution.

\section{How to cite this article}

Hilendarov A, Georgiev A, Chervenkov L. Invasive Procedures of the Chest Lesions- Are they must be Performed and Why. SSR Inst. Int. J. Life Sci., 2021; 7(3): 2807-2812.

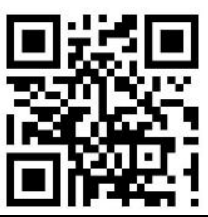

Indications of imaging-guided chest biopsy have significantly changed as a result of technical advances in needle types, imaging modalities, pathological analysis and immunohistological techniques. At present, the growing list of indications includes histological diagnosis of undetermined and otherwise not characteristically pulmonary, mediastinal and chest wall lesions as well as biopsy or re-biopsy of known malignant lesions to obtain histological material for targeted therapy ${ }^{[2]}$.

The use of MDCT has increased with the detection of small pulmonary nodules. In many patients, pulmonary nodules are incidentally detected in the general population ${ }^{[3]}$ in patients without clinical manifestation. If a nodule is initially identified at conventional chest radiography, CT investigation is necessary to establish the lesion, estimate the malignancy ${ }^{[4]}$ and identify lymphadenopathies or other accessible sites for biopsy extra-thoracic metastases ${ }^{[5]}$. PET-CT examination may 
also be helpful in the investigation of pulmonary nodules, reducing the need for puncture of nonenhancing solid nodules ${ }^{[6]}$. It should be borne in mind that active inflammation (i.e. active tuberculosis, histoplasmosis, rheumatoid nodules) may cause false positives on PET/CT scans because of their high glucose metabolism ${ }^{[7]}$. Although low-grade malignant tumours, such as carcinoid ${ }^{[8]}$ or low-grade adenocarcinoma ${ }^{[9]}$, may produce false-negative results because of their low glucose metabolism. In these patients, follow-up with CT must be performed to demonstrate regression of the nodule after therapy or if the increase in size is evident [10].

Mediastinal masses are most frequently located in the anterior mediastinum and include a variety of different entities, such as thymic malignancy, lymphomas, endocrine tumours and malignant germ cell tumours. In the absence of typical clinical and imaging features, histological diagnosis is necessary.

\section{MATERIALS AND METHODS}

Fine-needle aspiration biopsy (FNAB) and core-needle biopsy (CNB) currently is the predominant method for obtaining tissue specimens in patients with lung lesions. In many cases, treatment protocols are based on histological information. In 85 of all 97 patients included in our study, performed at the University Hospital in Dept of Diagnostic Imaging, FNAB biopsy is performed and in 12 patients(CNB), when technically feasible, or in cases where other techniques (such as bronchoscopy with lavage) are inconclusive. The 19-22G disposable needles were used.

Imaging guidance methods we used to guide chest biopsies. We have preferred CT guidance [11-15]. Parameters affecting the selection of the most appropriate imaging technique are the size and visibility of the lesion as well as its relationship with critical anatomical structures. Whenever possible, chest biopsies should be performed under US guidance to use the advantages of real-time monitoring without radiation to patients and operators ${ }^{[16]}$.

US guidance is limited to superficial lesions adjacent to the chest wall or to lesions delineated by pleural effusion sufficient to create a suitable interface for ultrasound penetration. In the significant majority of cases, MDCT is the preferred guidance method for chest biopsies due to its optimal spatial and contrast resolution. An intravenous contrast agent can be used to differentiate target lesions from atelectasis, necrosis and vascular structures.

The patient should be positioned prone, supine or lying on the side, based on the previously planned access site. Whenever possible, the needle access site should be cephalic to the ribs to avoid intercostal vessel and nerves puncture. The skin in the access site should be sterilized with an antiseptic solution and subcutaneous tissues should be infiltrated with lidocaine. Breath-hold capabilities are extremely different. It is easier to target larger tumours $(>2 / 3 \mathrm{~cm})$ instructing the patient to breathe freely with shallow respiration. In the remaining cases, the patient can be instructed to maintain an inspiratory or expiratory apnoea to allow easier access to target lesions.

Needle choice is based on the size and location of the lesion, planned needle pathway, status of the lung parenchyma and operator preference. Some of the used fine-needle aspiration devices include Chiba, Westcott, Greene (cook) and turner (cook) needles, which have circumferentially sharpened tips allowing for sampling.

Core biopsy needles are designed to collect a small piece of tissue intended for surgical pathology analysis and can be designed as end-cutting or side-cutting devices.

The most commonly used core biopsy needle is the TruCut, which consists of an outer cutting cannula and an inner slotted stylet. The presence of an on-site pathologist may reduce the number of biopsies needed.

\section{RESULTS}

In 76 patients, aged 21-79 years with pulmonary lesions with dimensions of $2.0 \mathrm{~cm}$ or less FNB under CT control are performed. In 13 cases FNA under US control is performed due to the superficial localization of the lesions (Fig. 1). Cytological and histological evaluation of FNAB samples were performed in all patients. Diagnostic sensitivity and accuracy are calculated. Assess the number and type of complications that occurred. Fineneedle aspiration biopsy (FNAB) and core-needle biopsy (CNB), with the latter, demonstrated to have a slightly higher overall sensitivity, specificity and accuracy.

We prefer MDCT for the guidance of FNB due to the better biopsy needle path control and the visibility of small-sized and deeply located lung lesions (Fig. 2). We have a wide variation in diagnostic accuracies of FNAB ranging from $64 \%$ to $97 \%$. 


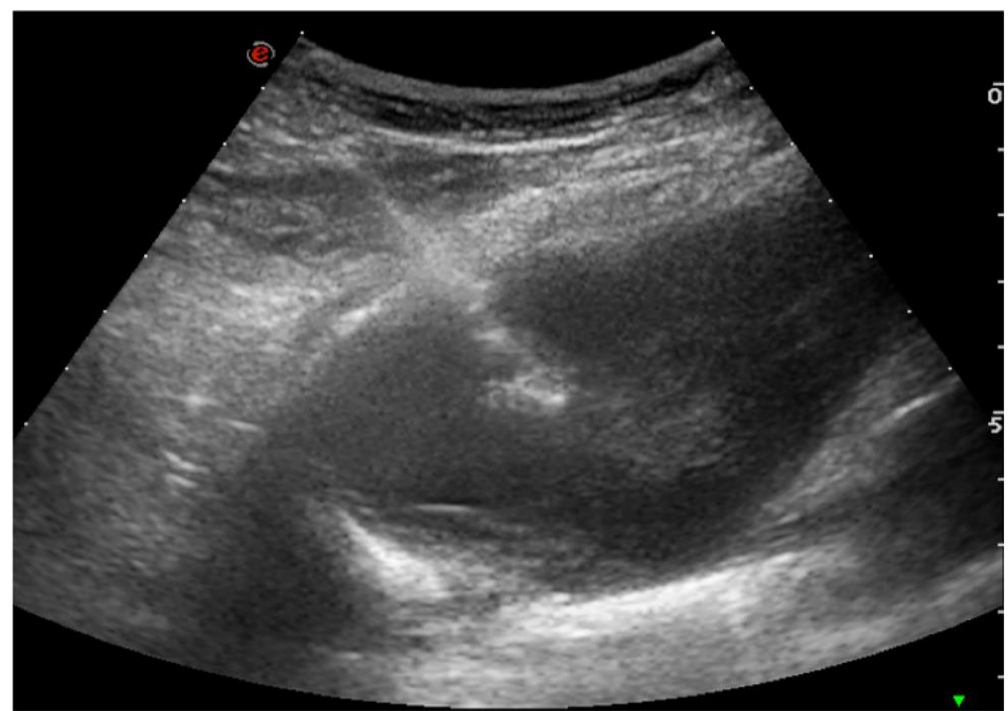

Fig. 1: Ultrasound-guided FNB biopsy of a pulmonary nodule in the left lower lobe. The needle was $20 \mathrm{G}$ due to suspicions of the liquid nature of the lesion
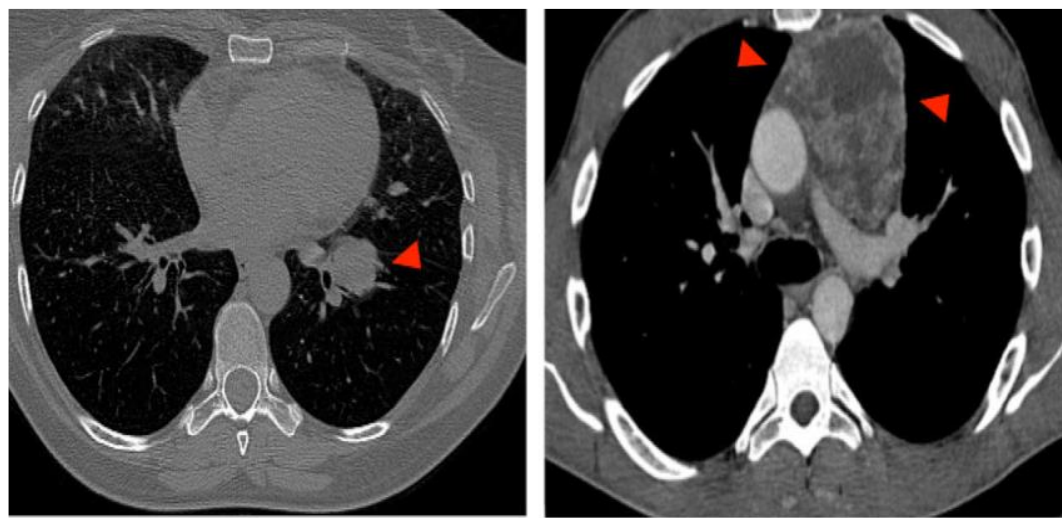

Fig. 2: CT guided biopsy. Indications for FNAB (a) Mass lesion following negative bronchoscopy (b) CNB of unknown mass in the anterior part of the mediastinum

Core biopsy has been shown to have slightly higher overall sensitivity, specificity and accuracy, with values of $89 \%, 97 \%$ and $93 \%$. The test of genetic mutations is important to plan targeted therapies in the patient with lung cancers. Therefore, it is necessary to obtain a sufficient amount of tissue sample (Fig. 3).

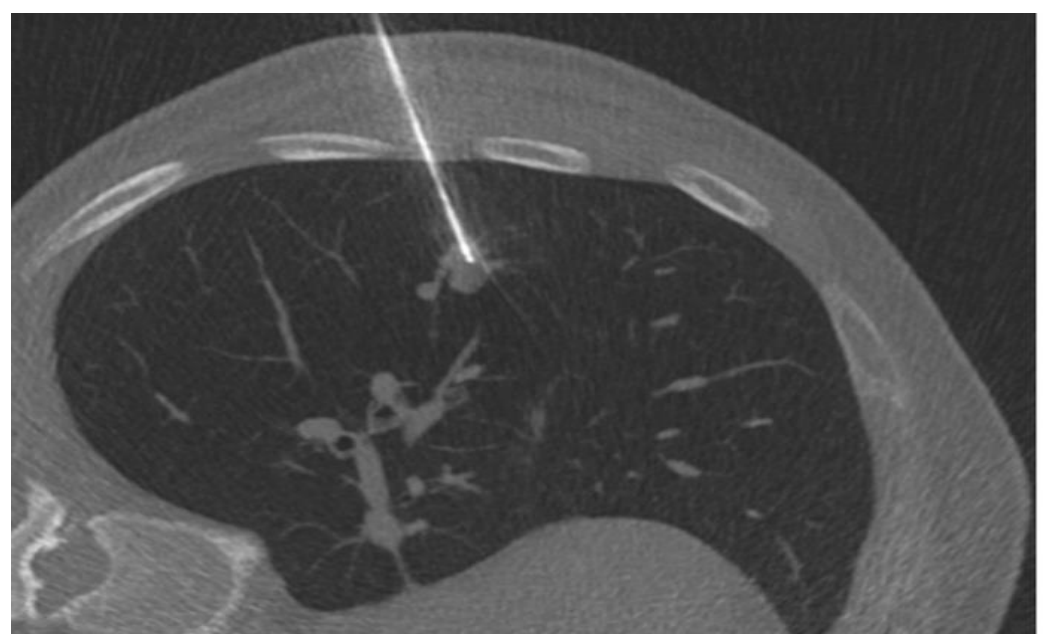

Fig. 3: CT image presenting left pulmonary lobe. CT-guided Core chest biopsy of a pulmonary nodule in the left lower lobe 
PNX occurs during or immediately after the procedure and can be detected on postprocedural control scans Fig. 4). PH may occur with or without haemoptysis and can be easily detected on screening post-biopsy CT scan as perilesional or needle tract ground-glass opacity (Fig. 5). In our patients, the effects of lesion location on the diagnostic accuracy of imaging-guided biopsy in chest tumours were evaluated.
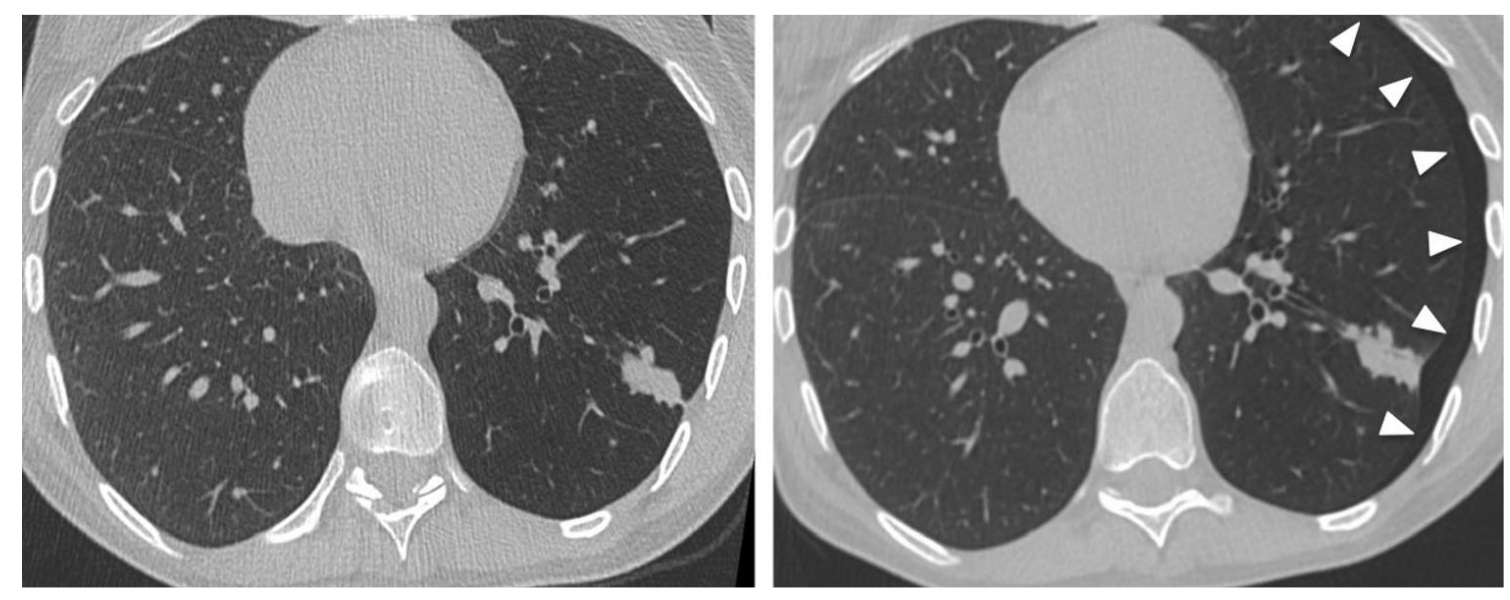

Fig. 4: CT-guided chest biopsy (a) Axial CT image before the procedure showing the left subpleural nodule; (b) Postprocedural axial CT image showing small PNX (arrows) as a complication of transthoracic
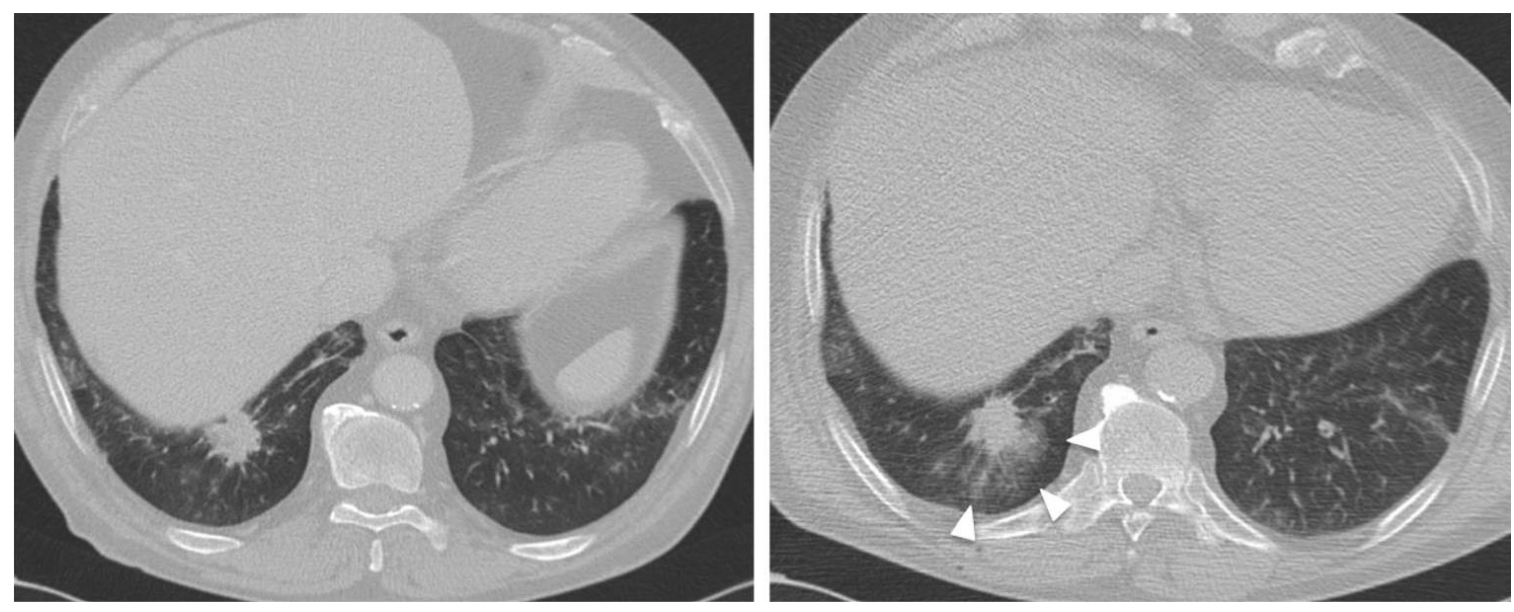

Fig. 5: CT-guided chest biopsy of a pulmonary nodule (a) Axial CT image showing a pulmonary nodule in the right lower lobe; (b) Postprocedural CT image demonstrates perilesional haemorrhage as ground-glass opacity around the nodule (arrows)

\section{DISCUSSION}

In literature reports, the sensitivity in diagnosing lung tumours decreased from $100 \%$ in peripheral lesions to $82 \%$ in central lesions. Wang et al. ${ }^{[14]}$ recently compared the rates of complications and diagnostic accuracy of CTguided biopsy in peripheral versus paramediastinal lesions, demonstrating how this technique may be safe and reliable even for deeply located tumours; in particular, their paper reports diagnostic accuracy of 95.4\% in paramediastinal lesions and $94.7 \%$ in peripheral lesions with a sensitivity of $95.6 \%$ and $94.2 \%$, respectively.
Once the biopsy has been performed, we perform control by $\mathrm{CT}$ of the chest is obtained to identify any immediate post-procedural complications ${ }^{[10]}$. According to some authors, the patients should be rolled over onto the punctured side to reduce the risk of delayed PNX; Chest films are usually acquired after $4 \mathrm{~h}$ to detect possible asymptomatic PNX. If the clinical suspicion of a PNX arises, chest radiography must be obtained immediately.

Pneumothorax (PNX) is the most common complication after imaging-guided chest biopsy; it is most frequently detected after lung biopsy but can also occur even after biopsies of mediastinal, pleural and chest wall lesions ${ }^{[11]}$. 
The incidence of PNX has been reported to be up to $61 \%$ with an average risk of $20 \%$. Risk factors for PNX can be related to patient or lesions features, but also the biopsy technique. In particular, the rate of PNX increases with the patient age and severity of underlying lung disease (e.g. emphysema or chronic obstructive disease) as well as in smaller and deeper lesions ${ }^{[16]}$. Technical risk factors include the type and size of the biopsy needle, longer procedure duration, and biopsies in the middle or lower lobe, transgression of a fissure and multiple needle repositioning or pleural passes ${ }^{[15]}$. A PNX developed during the procedure can be immediately aspirated through the introducer needle or a separate needle inserted into the pleural space, preventing further enlargement; however, some authors suggest placing a chest tube if aspirated air is greater than $670 \mathrm{ml}$. PNX can be significant ( $>30 \%$ of lung volume), increase over time or become symptomatic. In these cases, small-calibre, 6to 9-French catheters can be safely and easily placed.

Haemorrhage represents the second most common complication after imaging-guided biopsy ${ }^{[8]}$. The occurrence rates of $\mathrm{PH}$ are estimated to be from 4 to $27 \%$ (with an average incidence of $11 \%$ ), while haemoptysis risk is up to $5^{[9]}$. Complication does not need any treatment and the only recommendations are to place the patient in a lateral position, with the biopsy side down, to avoid aspiration of blood into the unaffected lung. Occasionally a larger, higher-grade PH occurs and pro-coagulative treatment may be needed. Risk factors for higher-grade $\mathrm{PH}$ include older patients with emphysema, pulmonary hypertension, coaxial technique, sub-solid lesions, and lesion size smaller than $3 \mathrm{~cm}{ }^{[17]}$. The occurrence of systemic air embolism (SAE) in the left atrium, left ventricle or systemic circulation is rare (incidences between $0.01 \%$ and $0.21 \%$ ), but potentially fatal cardiac infarct. There are three mechanisms in particular responsible for SAE during biopsy: placement of the needle tip in a pulmonary vein, formation of a bronchial-venous or alveolar-venous fistula and opening the outer cannula of a coaxial biopsy needle to the atmosphere.

Tumour seeding through the needle track represents a very rare complication with a prevalence reported in the literature between 0.012 and $0.061 \%$. The real clinical relevance is still discussed, but it is obvious that tumour seeding along the needle track can significantly change patient management and life expectancy and should be strictly avoided ${ }^{[9]}$. Tumour seeding is reported to be more frequently observed after imaging-guided core needle biopsy of pleural mesothelioma.

\section{CONCLUSIONS}

In conclusion, imaging-guided chest biopsy is an interventional procedure of pivotal importance for several clinical conditions of pneumatological, oncological and surgical interest.

This procedure may appear very simple, but radiologists approaching it for the first time must consider several clinical and technical variables significantly affecting the final results, in terms of both diagnostic accuracy and patients' safety.

\section{CONTRIBUTION OF AUTHORS}

Research concept- Dr. Atanas Hilendarov

Research design- Alexandar Georgiev

Supervision- Dr. Atanas Hilendarov

Materials- Alexandar Georgiev

Data collection- Lyubomir Chervenkov

Data analysis and Interpretation- Dr. Atanas Hilendarov

Literature search- Alexandar Georgiev

Writing article- Alexandar Georgiev

Critical review- Lyubomir Chervenkov

Article editing- Dr. Atanas Hilendarov

Final approval- Dr. Atanas Hilendarov

\section{REFERENCES}

[1] Viggiano RW, Swensen SJ, Rosenow EC $3^{\text {rd }}$ Evaluation and management of solitary and multiple pulmonary nodules. Clin Chest Med., 1992; 1: 83-95.

[2] Hammerschlag G, Cao J, Gumm K, Irving L, Steinfort $\mathrm{Db}$ Prevalence of incidental pulmonary nodules on computed tomography of the thorax in trauma patients. Intern Med J., 2015; 6: 630-33.

[3] Laroche C, Fairbairn I, Moss H, Pepke-Zaba J, Sharples $L$, et al. Role of computed tomographic scanning of the thorax prior to bronchoscopy in the investigation of suspected luncance Thorax55 https://insightsimaging.springeropen.com/articles/1 0.1007/s13244-017-0561-6(2000; 5: 359-63.

[4] Erasmus JJ, McAdams HP, Connolly JE. Solitary pulmonary nodules: part II. Evaluation of the indeterminate nodule. Radiographics, 2000; 1: 5966.

[5] MacMahon H, Austin JH, Gamsu G, Herold CJ, Jett JR, et al. Guidelines for management of small pulmonary 
nodules detected on CT scans: a statement from the Fleischner Society. Radiol., 2005; 2: 395-400.

[6] Nosari A, Anghilieri M, Carrafiello G, Guffanti C, et al. Utility of percutaneous lung biopsy for diagnosing filamentous fungal infections in hematologic malignancies. Haematologica., 2003; 12: 1405-09.

[7] Lange TJ, Kunzendorf F, Pfeifer M, Arzt M, Schulz C. Endobronchial ultrasound-guided transbronchial needle aspiration in routine care - plenty of benign results and follow-up tests. Int J Clin Pract., 2012; 5: 438-45.

[8] Carter BW, Okumura M, Detterbeck FC, Marom E. Approaching the patient with an anterior mediastinal mass: a guide for radiologists. J Thorac Oncol., 2014; 9 Suppl 2): S110-S18.

[9] Laurent F, Montaudon $M$, Latrabe $V$, Bégueret $H$. Percutaneous biopsy in lung cancer. Eur J Radiol., 2003; 1: 60-68.

[10]Winokur RS, Pua BB, Sullivan BW, Madoff DC. Percutaneous lung biopsy: technique, efficacy, and complications. Semin Intervent Radiol., 2013; 2: 12127.

[11]DiBardino DM, Yarmus LB, Semaan RW. Transthoracic needle biopsy of the lung. J Thorac Dis., 2015; Suppl 4: S304-S316.
[12]Schneider F, Smith MA, Lane MC, et al. Adequacy of core needle biopsy specimens and fine-needle aspirates for molecular esting of lung adenocarcinomas. Am J Clin Pathol., 2015; 2: 193200.

[13]Wang $Y$, Jiang F, Tan X, Tian P. CT-guided percutaneous transthoracic needle biopsy for paramediastinal and nonparamediastinal lung lesions: diagnostic yield and complications in 1484 patients. Medicine, 2016; 31: e4460.

[14]Anzidei M, Sacconi B, Fraioli F, Saba L, et al. Development of a prediction model and risk score for procedure-related complications in patients undergoing percutaneous computed tomographyguided lung biopsy. Eur J Cardiothorac Surg., 2015; 1: e1-e6.

[15]Geraghty PR, Kee ST, McFarlane G, et al. CT-guided transthoracic needle aspiration biopsy of pulmonary nodules: needle size and pneumothorax rate. Radiol., 2003; 229: 475-81.

[16]Henry M, Arnold T, Harvey J. BTS guidelines for the management of spontaneous pneumothorax. In: BTS guidelines for the management of pleural disease. Thorax, 2003; Suppl II: ii39-ii52.

[17]Hare SS, Gupta A, Goncalves AT, Souza CA, Matzinger $F$, et al. Systemic arterial air embolism after percutaneous lung biopsy. Clin Radiol., 2011; 7: 58996.

\section{Open Access Policy:}

Authors/Contributors are responsible for originality, contents, correct references, and ethical issues. SSR-IIJLS publishes all articles under Creative Commons Attribution- Non-Commercial 4.0 International License (CC BY-NC). https://creativecommons.org/licenses/by-nc/4.0/legalcode (c) (1) (9) 